\title{
Patient-Reported Outcome Measures Used for Assessing Breast Sensation after Mastectomy: Not Fit for Purpose
}

\author{
Hansje P. Smeele ${ }^{1} \mathbb{1} \cdot$ Rachel C. H. Dijkstra ${ }^{1} \cdot$ Merel L. Kimman $^{2}{ }^{(1)} \cdot$ René R. W. J. van der Hulst $^{1}$. \\ Stefania M. H. Tuinder ${ }^{1} \mathbb{C}$
}

Accepted: 29 November 2021 / Published online: 18 January 2022

(c) The Author(s) 2022

\begin{abstract}
AIMS The aims of this review were (i) to evaluate whether patient-reported outcome measures used in clinical studies for assessing sensation after mastectomy and breast reconstruction are suitable for this purpose, and (ii) to explore whether any measures used for assessing sensation after non-oncologic breast surgery are worth modifying for use in post-mastectomy patients.

Methods PRISMA guidelines were followed (PROSPERO number CRD42020178066). We searched six databases for studies of oncologic (i.e., therapeutic, prophylactic, and reconstructive) and non-oncologic breast surgery (e.g., breast reduction) in which sensation was assessed with a patient-reported outcome measure. From the selected studies, we extracted eligible measures, evaluated their fitness for purpose, and summarized the content of sensation-specific items.

Results Of 6728 articles identified, we selected 135 studies that used 124 eligible patient-reported outcome measures. For $97 \%$ of these measures, details regarding development and measurement properties were unavailable. Four (3\%) validated measures-the Sensory Disturbances subscale of the Breast Cancer Sequelae Cause Scales, the Discomfort subscale of the Breast Sensation Assessment Scale (BSAS), Didier et al.'s questionnaire for "Assessment of the patients' satisfaction with cosmetic results, physical and emotional impact of mastectomy", and the Breast Specific Pain subscale of the Breast Cancer Treatment Outcomes Scale (BCTOS)—each contain at least one item pertaining to breast sensation, but target different concepts of interest. In total, the measures feature 215 sensation-specific items, most of which concern symptom severity (97\%) as opposed to impact on daily functioning (3\%).

Conclusion Patient-reported outcome measures used in clinical studies for assessing sensation after mastectomy and breast reconstruction are unsuitable for this purpose: they are either non-validated or non-specific. We failed to identify any measures for use in non-oncologic breast surgery populations worth modifying. To collect meaningful, patient-relevant data regarding sensation after mastectomy, it is pertinent that future clinical trials adopt psychometrically robust, specific patient-reported outcome measures.
\end{abstract}

\section{Key Points for Decision Makers}

Hansje P. Smeele and Rachel C. H. Dijkstra have made an equal contribution to the work.

Stefania M. H. Tuinder

s.tuinder@mumc.nl

1 Department of Plastic, Reconstructive and Hand Surgery, GROW, School for Oncology and Developmental Biology, Maastricht University Medical Center, P.O. Box 5800, 6202 AZ Maastricht, The Netherlands

2 Department of Clinical Epidemiology and Medical Technology Assessment, Care and Public Health Research Institute (CAPHRI), Maastricht University Medical Center, P.O. Box 5800, 6202 AZ Maastricht, The Netherlands
Patient questionnaires can inform us about added value of treatments aimed at preserving or restoring normal sensation after breast surgery.

This systematic review shows that patient questionnaires previously used for evaluating loss and regain of breast sensation are not fit for this purpose: their quality is unknown, or they evaluate outcomes broader than breast sensation alone.

To obtain valid results, future studies should adopt high-quality patient questionnaires that focus on breast sensation alone. 


\section{Introduction}

After mastectomy and breast reconstruction for (pre-)malignant disease, patients are often confronted with loss of normal skin sensation [1, 2]. For many women, the breast and nipple are erogenous zones [3], and reduced sensation may negatively affect their sexual functioning. Tactile feedback could be an important mechanism by which patients recognize their reconstructed breast as part of their body, as is the case in hands [4]. In addition, reduced ability to sense heat predisposes reconstructed breasts to (severe) burns [5-8].

Growing recognition of the importance of breast sensation and adverse effects of loss of sensation have encouraged innovations to enhance sensory outcomes. Examples include skin- and nipple-sparing mastectomy, and 'innervated' breast reconstruction through surgical connection of sensory nerves or implantation of electronic sensors [9-11].

Loss or gain of injury-preventing sensation can be assessed through quantitative sensory testing [12]. But for evaluating whether attempts to preserve or restore sensation fulfill patient needs, asking them directly provides the only reasonable strategy. Patient-reported outcome measures ${ }^{1}$ do exactly this: they capture the patient's perception of achieved results. They can focus on symptoms (e.g., pain, fatigue), functional outcomes (e.g., physical, sexual), or broader concepts such as health-related quality of life, and are increasingly included as trial endpoints to inform clinical practice and policy [13].

Three systematic reviews summarizing outcomes of innervated autologous breast reconstruction signaled a variety of patient-reported outcome measures used for assessing sensation [14-16], indicating absence of a standardized approach. The identified measures were not further described, let alone critically appraised. To guide future clinical trials toward selecting well-suited measures, we aimed (i) to evaluate whether patient-reported outcome measures used in clinical studies for assessing sensation after mastectomy and breast reconstruction are suitable for this purpose, and (ii) to consider whether sensation-specific measures used in studies of non-oncologic breast surgery populations are worth modifying for use in post-mastectomy patients.

\section{Methods}

\subsection{Protocol and Registration}

This systematic review was conducted in adherence to the Preferred Reporting Items for Systematic Reviews and MetaAnalyses (PRISMA) guidelines [17]. The review protocol

\footnotetext{
1 Terms italicized on first use in the text are defined in the glossary (Online Resource 1, see electronic supplementary material [ESM]).
}

was registered with the International Prospective Register of Systematic Reviews (PROSPERO), and is available at www. crd.york.ac.uk/PROSPERO, CRD42020178066.

\subsection{Working Definition of Breast Sensation}

To the best of our knowledge, there exists no agreed definition for the concept of breast sensation. We created and used a working definition comprising

- ability to feel sensory stimuli (e.g., touch, pressure, vibration, temperature, and pain) in the breast, the nipple, or both;

- ability to experience affective aspects of touch (e.g., pleasant, sexual) in the breast, the nipple, or both;

- ability of the nipple to become erect upon a sensory stimulus (e.g., cold, touch);

- symptoms indicating end-organ sensory loss (e.g., hypoesthesia, numbness, and anesthesia) in the breast, the nipple, or both.

The way the breast feels to the touch (e.g., texture, softness), and symptoms resulting from abnormal somatosensory processing (e.g., dysesthesia, paresthesia, hyperalgesia, allodynia, spontaneous pain, and phantom sensations) [18] fall outside this definition.

\subsection{Search Strategy}

In consultation with a librarian, we developed a search strategy [19] with controlled vocabulary and relevant keywords linked to three pillars: (i) breast or nipple surgery, (ii) sensation, and (iii) patient-reported outcome assessment (Online Resource 2, see ESM). Modifying and validating measures developed for a related population of interest can increase efficiency and opportunities for comparison [20]. Therefore, we searched for studies of oncologic (i.e., therapeutic, prophylactic, and reconstructive) as well as non-oncologic surgery (e.g., breast reduction). We searched six electronic databases (Medline [PubMed], Embase, CINAHL, Web of Science, Cochrane Library, and Google Scholar) for articles published before April 30, 2020. Database searches were supplemented by a manual backward reference check of systematic reviews summarizing sensory outcomes after various types of breast surgery. The United States National Library of Medicine clinical trials registry (ClinicalTrials. gov) was searched for studies in progress.

\subsection{Study and Patient-Reported Outcome Measure Selection}

We included peer-reviewed clinical studies of breast or nipple surgery if a patient-reported outcome measure was used 
to assess sensation. Conference abstracts, case reports, qualitative studies, and articles published in languages other than English, German, and French were excluded. After removing duplicates, H.P.S. and R.C.H.D. independently screened titles and abstracts of all studies, followed by retrieval and assessment of full-text articles. Studies unavailable in full text were excluded. Disagreements were resolved through discussion with M.L.K. From the selected studies, we identified all patient-reported outcome measures used for assessing breast sensation. Incompletely presented measures that could not be obtained from the corresponding author were excluded, as were measures without at least one item pertaining to breast sensation as defined in Sect. 2.2.

\subsection{Data Extraction}

The data extraction protocol was tested in five eligible articles and revised accordingly. H.P.S. extracted study- and measure-specific information (e.g., country of origin, study design, characteristics of study population, time elapsed from surgery to survey, concept of interest, sensation-specific items with response options, whether the concept of breast sensation makes up an independent score, information regarding development and measurement properties [Online Resource 3, see ESM]) into a Microsoft Excel spreadsheet. R.C.H.D. cross-checked data extracted from a random subset of 20 articles for accuracy and completeness.

\subsection{Patient-Reported Outcome Measure Appraisal}

We considered measures suitable for the purpose of assessing sensation after mastectomy and breast reconstruction, or worthy of adaptation for this purpose, (i) if they generate an independent score for the concept of breast sensation, and (ii) if they are valid and reliable according to the COnsensusbased Standards for the selection of health Measurement INstruments) methodology [21]. Measures that do not meet these criteria were considered unsuitable, as were measures for which information regarding development and measurement properties was unavailable. Quality and risk of bias of primary studies exceeded the scope of this review.

\subsection{Item Content Synthesis}

We compared sensation-specific items, looking for similarities regarding content and inductively grouping similar items into categories to form an affinity diagram [22]. Consensus on competing interpretations was achieved through discussion with M.L.K.

\section{Results}

\subsection{Study and Patient-Reported Outcome Measure Selection}

Our systematic search of the literature yielded 8563 citations, of which 6728 remained after deduplication. From 135 studies deemed relevant, we identified 124 eligible unique patient-reported outcome measures (Fig. 1).

\subsection{Study Characteristics}

Approximately half the included studies $(51.1 \%)$ focused on oncologic breast surgery; the other half concerned nononcologic procedures (Table 1 and Online Resource 4, see ESM). In $16.3 \%$ of studies, breast sensation was the primary endpoint. Few studies (12.6\%) were prospective in design, whilst the vast majority (87.4\%) used a cross-sectional design (i.e., patients completed the measure at just one postoperative follow-up moment). Additional quantitative sensory testing was performed in $<30 \%$ of studies.

\subsection{Patient-Reported Outcome Measure Characteristics}

We identified four patient-reported outcome measures (3\%) for which details regarding development and measurement properties were available: the Sensory Disturbances subscale of the Breast Cancer Sequelae Cause Scales [33], the Discomfort subscale of the Breast Sensation Assessment Scale (BSAS) [34], Didier et al.'s questionnaire for "Assessment of the patients' satisfaction with cosmetic results, physical and emotional impact of mastectomy" [35], and the Breast Specific Pain subscale of the Breast Cancer Treatment Outcomes Scale (BCTOS) [36] (Fig. 2). These measures contain only one or three items related to breast sensation, and their summary scores represent different concepts of interest. The remaining 97\% concerned ad hoc measures that were not validated, or only minimally (e.g., readability pilot test) [37-40]. Most ad hoc measures (92\%) were used in a single study (Fig. 2). In six studies, items from existing patient-reported outcome measures were compiled into a new study-specific measure [39-44], whilst in the remaining studies, methods for item content generation were rarely described. Authors who used multi-item ad hoc measures often disaggregated sensation-specific item responses and analyzed them as a standalone score. The average number of sensation-specific items per measure was 1.7 (range 1-7). 


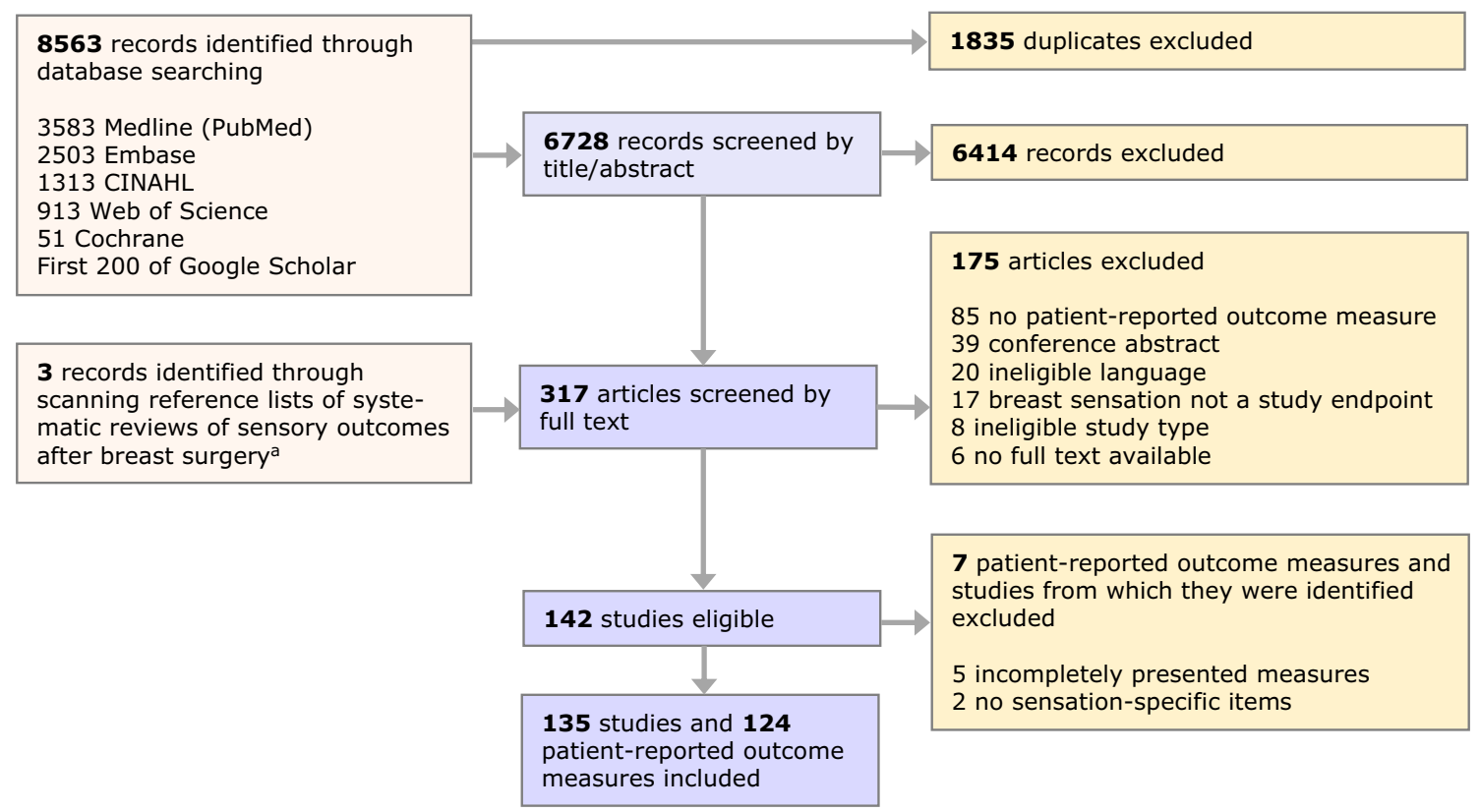

Fig. 1 PRISMA flow diagram of the study and patient-reported outcome measure selection process ${ }^{a}$ Reference lists of eight systematic reviews $[2,15,16,23-27]$ were scanned

\subsection{Suitability of Measures}

Since none of the identified validated measures generate an independent score for the concept of breast sensation, we considered them unsuitable for the purpose of assessing this outcome. The ad hoc measures were deemed unsuitable or unworthy of modification given the lack of available details regarding development and measurement properties.

\subsection{Item Content}

In total, the various measures contained 215 individual items related to breast sensation. Compilation of these items revealed similarities regarding the aspect of breast sensation evaluated (e.g., erogenous sensation), question type (i.e., symptom severity versus impact on daily functioning), and anatomical area (i.e., nipple versus the whole breast; Fig. 3 and Online Resource 4, see ESM). Most items investigated symptom severity (97\%) and were framed in general terms (e.g., "To what extent is your sensation in the breast area reduced?" [45]) rather than in the context of everyday situations (e.g., "Do you experience any kind of sensibility in your reconstructed breast when $[\ldots]$ taking a shower $[\ldots]$ ?" $[1,46])$. Only $3 \%$ of items explored the perceived impact of breast sensation —or a lack thereof-on everyday (mostly sexual) functioning.

\section{Discussion}

In this systematic review, we set out to describe patientreported outcome measures used in clinical studies for assessing breast sensation. We found that (i) measures used in studies of mastectomy and breast reconstruction are unsuitable for this purpose, and (ii) measures used in studies of non-oncologic breast surgery are not worth modifying and validating for use in post-mastectomy patients. We identified two main categories of measures, inadequate for different reasons: (i) homegrown ad hoc measures of unknown validity and reliability, and (ii) validated measures that were intended to assess concepts of interest broader than-and sometimes unrelated to-breast sensation. What is lacking is a psychometrically robust, content-specific measure.

A crucial flaw of ad hoc measures is the lack of patient involvement during development and testing [47]. Measures based primarily on sources other than the target population cannot be assumed to address their priorities and concerns, and as such, they are unsuitable for informing us about the patient benefit of an intervention. Although few authors of included ad hoc measures stated the methods used to generate content, items themselves suggest a great deal about the degree of patient consultation. Examples include frequent use of medical vocabulary that patients may find difficult to understand (e.g., areola, erogenous, tactile), the quantitative nature of items (e.g., "percentage change from the preoperative situation" [48]), or their dubious relevance (e.g., "satisfaction with nipple sensation in job-related situations" [39]). 
Table 1 Characteristics of included studies $(n=135)$

\begin{tabular}{|c|c|}
\hline & No. (\%) of studies \\
\hline \multicolumn{2}{|l|}{ Publication year } \\
\hline$\leq 1994$ & $13(10)$ \\
\hline 1995-1999 & $14(10)$ \\
\hline $2000-2004$ & $20(15)$ \\
\hline 2005-2009 & $21(16)$ \\
\hline 2010-2014 & $36(27)$ \\
\hline 2015-April 2020 & $31(23)$ \\
\hline \multicolumn{2}{|l|}{ Geographic origin } \\
\hline Europe & $72(53)$ \\
\hline North America & $39(29)$ \\
\hline Asia & $15(11)$ \\
\hline Africa/South America/Australia & $9(7)$ \\
\hline \multicolumn{2}{|l|}{ Study design } \\
\hline Cross-sectional study & $118(87)$ \\
\hline Prospective cohort study & $13(10)$ \\
\hline Randomized controlled trial & $4(3)$ \\
\hline \multicolumn{2}{|l|}{ Breast surgery type ${ }^{a}$} \\
\hline $\begin{array}{l}\text { Oncologic (therapeutic/prophylactic/reconstruc- } \\
\text { tive) }\end{array}$ & $69(51)$ \\
\hline Breast-conserving surgery & $10(7)$ \\
\hline Mastectomy & $64(47)$ \\
\hline Skin-sparing mastectomy & $43(32)$ \\
\hline Nipple-sparing mastectomy & $16(12)$ \\
\hline Breast reconstruction & $62(46)$ \\
\hline (Expander-)implant & $41(30)$ \\
\hline Free flap ${ }^{\mathrm{b}}$ & $38(28)$ \\
\hline Latissimus dorsi flap + implant & $7(5)$ \\
\hline Nipple reconstruction $^{\mathrm{c}}$ & $15(11)$ \\
\hline Non-oncologic & $66(49)$ \\
\hline Breast reduction/mastopexy & $44(33)$ \\
\hline Breast augmentation & $19(14)$ \\
\hline Lactiferous duct excision & $2(2)$ \\
\hline Nipple reduction/inverted nipple correction & $2(2)$ \\
\hline \multicolumn{2}{|l|}{ Breast sensation as study endpoint } \\
\hline Primary study endpoint & $22(16)$ \\
\hline Secondary study endpoint & $113(84)$ \\
\hline \multicolumn{2}{|l|}{ Sample size } \\
\hline $10-49$ & $46(34)$ \\
\hline $50-99$ & $29(22)$ \\
\hline $100-149$ & $24(18)$ \\
\hline$\geq 150$ & $36(27)$ \\
\hline \multicolumn{2}{|l|}{ Time elapsed from surgery to survey ${ }^{\mathrm{a}}$} \\
\hline Preoperative & $2(2)$ \\
\hline$<12$ months & $18(13)$ \\
\hline$\geq 12$ months & $86(64)$ \\
\hline Unknown & $29(22)$ \\
\hline \multicolumn{2}{|l|}{ Additional objective sensory testing } \\
\hline No & $96(71)$ \\
\hline Yes $^{\mathrm{a}}$ & $39(29)$ \\
\hline Mechanical detection threshold & $38(28)$ \\
\hline
\end{tabular}

Table 1 (continued)

\begin{tabular}{ll}
\hline & No. (\%) of studies \\
\hline Thermal detection threshold (warmth/cold) & $14(10)$ \\
Pain threshold (pressure/heat) & $10(7)$ \\
Vibration detection threshold & $5(4)$ \\
Nipple erectility & $5(4)$ \\
Other & $7(5)$ \\
\hline
\end{tabular}

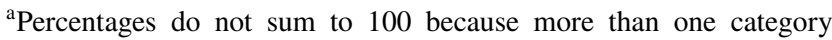
could apply to a study

${ }^{\mathrm{b}}$ Includes five studies of innervated free tissue flaps [28-32]

${ }^{\mathrm{c}}$ Only studies that specifically evaluated outcomes of nipple reconstruction were counted

Unavailability of a patient-reported outcome measure suitable for the purpose of assessing breast sensation may have compelled authors to develop ad hoc measures specifically for their studies. Until the Breast-Q was introduced in 2009 , this practice was common in the wider reconstructive and cosmetic breast surgery field [49]. Creating ad hoc measures is a pragmatic short-term fix, but leads to a proliferation of new, overlapping measures. This is unhelpful, both because the quality of data being collected is uncertain, and because the unmet need-a robust, specific measureremains unaddressed.

To ensure that measures cover those aspects of concern to patients, their content should be informed by an openended understanding of what breast sensation means to them. Qualitative studies have previously touched upon patients' perceptions regarding loss of normal sensation after lumpectomy [50], mastectomy alone [51], and postmastectomy breast reconstruction [50-58], providing relevant insights, but failing to cover the concept in detail. Our own team interviewed healthy women to establish a holistic frame of reference for understanding breast sensation [59]. The themes covered in this framework go beyond physical sensations of numbness, touch, and temperature that are the primary focus of patient-reported outcome measures identified by this review (Fig. 3). This discrepancy between findings highlights the need for further qualitative work elucidating which aspects of sensation are relevant to patients operated on for (pre-)malignant breast disease.

We identified four validated measures: the Sensory Disturbances subscale of the Breast Cancer Sequelae Cause Scales [33], the Discomfort subscale of the Breast Sensation Assessment Scale (BSAS) [34], Didier et al.'s questionnaire for "Assessment of the patients' satisfaction with cosmetic results, physical and emotional impact of mastectomy" [35], and the Breast Specific Pain subscale of the Breast Cancer Treatment Outcomes Scale (BCTOS) [36]. These are unsuitable for assessing breast sensation because their summary scores represent a different concept of interest. Other 


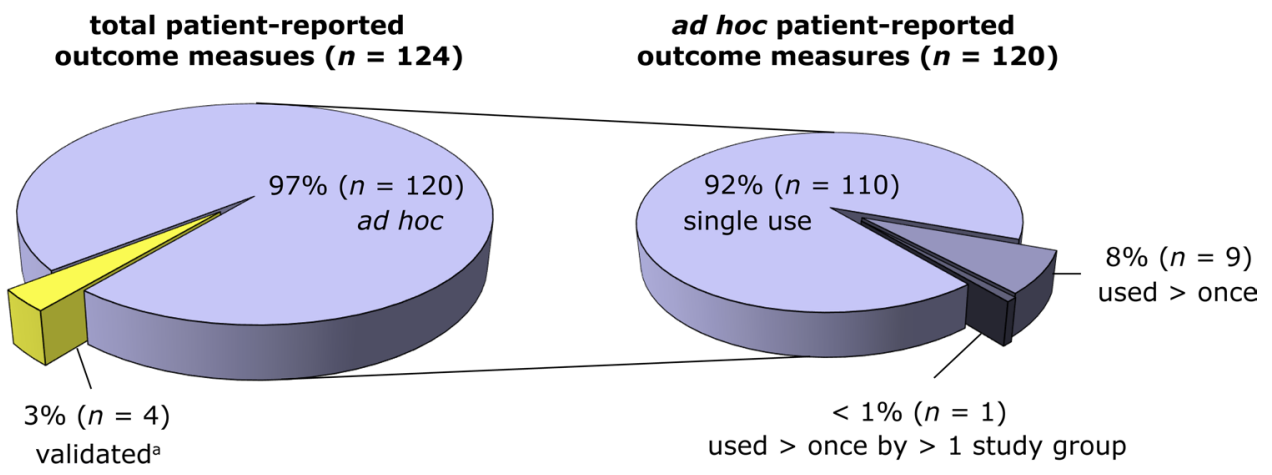

Fig. 2 Characteristics of included patient-reported outcome measures $(n=124)$. The left pie chart represents the proportion of validated (yellow segment) versus ad hoc patient-reported outcome measures (purple segment); the right pie chart shows the number of ad hoc measures that were reused in multiple studies. ${ }^{\text {a Sensory Disturbances }}$ subscale of the Breast Cancer Sequelae Cause Scales [33], Discom- fort subscale of the Breast Sensation Assessment Scale (BSAS) [34], Didier et al.'s questionnaire for "Assessment of the patients' satisfaction with cosmetic results, physical and emotional impact of mastectomy" [35], and Breast Specific Pain subscale of the Breast Cancer Treatment Outcomes Scale (BCTOS) [36]

\begin{tabular}{|c|c|c|c|c|}
\hline \multicolumn{5}{|c|}{ BREAST SENSATION } \\
\hline \multicolumn{2}{|l|}{ 1. Aspect } & \multicolumn{2}{|l|}{$\begin{array}{l}\text { 2. Question type } \\
\text { Symptom severity }\end{array}$} & 3. Area \\
\hline Sensation & $134(62 \%)$ & Absolute & $90(42 \%)$ & Nipple \\
\hline Erogenous sensation & $27(12 \%)$ & $\begin{array}{l}\text { Example: "How would you rate the erogenous } \\
\text { sensation of your reconstructed breast?" (very } \\
\text { much/some/little/none) }\end{array}$ & & \\
\hline & & Comparative & $83(38 \%)$ & \\
\hline Touch sensation & $17(8 \%)$ & $\begin{array}{l}\text { Example: "Has your ability to feel sensation in the } \\
\text { nipple changed after your breast surgery?" (8-point }\end{array}$ & & \\
\hline Numbness & $11(5 \%)$ & $\begin{array}{l}\text { Likert scale from "have no sensation" to "unchanged } \\
\text { sensation") }\end{array}$ & & Bre \\
\hline Temperature sensation & $10(5 \%)$ & $\begin{array}{l}\text { Satisfaction or importance } \\
\text { Example: "How satisfied are you with skin sensitivity } \\
\text { in the breast?" (4-point Likert scale from }\end{array}$ & $36(17 \%)$ & $7 \%)$ \\
\hline Nipple erection & $8(4 \%)$ & "dissatisfied" to "very satisfied") & & \\
\hline & & Impact & $6(3 \%)$ & \\
\hline Pain sensation & $6(3 \%)$ & $\begin{array}{l}\text { Example: "Did sexual pleasure change since the } \\
\text { operation because of loss of nipple-areola complex }\end{array}$ & & \\
\hline Pressure sensation & $2(1 \%)$ & $\begin{array}{l}\text { sensitivity?" (5-point Likert scale from "absent" to } \\
\text { "unchanged") }\end{array}$ & & \\
\hline
\end{tabular}

Fig. 3 Affinity diagram showing categorized content of items related to breast sensation. The content of individual items pertaining to breast sensation $(n=215)$ was grouped on three different but equal levels: (1) the aspect of breast sensation evaluated (e.g., erogenous sensation); (2) question type: the patient's rating of symptom severity (in absolute terms, in comparison to before the operation or to the unoperated side, or satisfaction with or importance of breast sensa-

symptoms in the measure may blur subtle but potentially important changes in sensation caused by the intervention. In theory, sensation-specific items within these measures can be validated for independent scoring, though their narrow representation of the concept of breast sensation hardly justifies the effort.

Generic patient-reported outcome measures, which we excluded from this review altogether because they lack tion) versus the perceived impact of present or absent breast sensation on everyday functioning; and (3) anatomical area (i.e., nipple versus the whole breast) [22]. Data are $n(\%)$. Categories within each column are mutually exclusive and percentages sum to 100 . For an overview of individual items synthesized in this diagram, see Online Resource 4 in the ESM

breast sensation-specific items, raise a similar problem. In the only completed randomized controlled trial to date regarding the effect of innervated autologous breast reconstruction on quality of life [60], Temple and colleagues used three validated measures that were "too general to detect changes related to breast sensation in a responsive and meaningful manner", according to Alderman and Chung [61]. Stromps et al. studied spontaneous return of sensation 
after autologous breast reconstruction and used the generic SF-36 questionnaire to capture patient perspectives [62]. The authors refrained from drawing conclusions regarding patient-reported sensory outcomes because summary scores were likely influenced by factors beyond the intervention. To avoid this kind of problem, researchers are increasingly being encouraged to choose specific patient-reported outcomes (e.g., disease or intervention-specific symptoms), rather than broader concepts (e.g., health-related quality of life) as primary trial endpoints [13].

Large numbers of patients may benefit from a robust, sensation-specific measure. Breast cancer represents one in four cancer diagnoses among women globally [63]. In countries where breast reconstruction is relatively accessible-such as the Unites States, Australia, Japan, and several European countries - the non-weighted average uptake after therapeutic mastectomy is $17 \%$ [64]. Increasing numbers of women continue to live with the consequences of these surgeries owing to the rising incidence of breast cancer [65], a rapid increase in women with unilateral breast cancer electing bilateral mastectomy [66], and prolonged survival after diagnosis [67]. Special efforts are being made to preserve and restore these patients' normal physical, sexual, and social functioning as much as possible. In this context, addressing loss of normal skin sensation is becoming an important reconstructive goal. The abundance of studies identified by this review indicates a widespread desire to evaluate patient benefit as a result of these efforts, and underlines the need for a high-quality measure.

At least two measures developed in adherence to established methodological standards have recently or will soon become available: (i) the Breast-Q Return of Sensation [68], and (ii) Abramsohn and colleagues' PROMIS measure for breast function recovery after mastectomy in relation to women's sexual functioning [69]. These measures have not yet been implemented in clinical research, and fell outside our eligibility criteria. However, with the advent of betterquality alternatives, there will soon be no reason to remain with ad hoc or ill-targeted measures in this field.

A limitation of our review is that the included measures did not allow for critical appraisal of their validity and reliability following the COSMIN methodology [21]. The ad hoc measures were characterized by an overall unavailability of details concerning development, impeding any assessment; the validated measures were off-topic and appraising their measurement properties would not have served the purpose of this review. Nonetheless, the available data allow us to conclude that studies have so far failed to assess patientreported breast sensation in a methodologically rigorous way. This has not been highlighted before.

\section{Conclusion}

This systematic review demonstrates that patient-reported outcome measures used in clinical studies for assessing breast sensation are unfit to do the job. To gather sound data that can be compared within and across studies, it is paramount that rigorously developed measures be adopted. These measures should be specific enough to elucidate the real effects of an intervention and changes during a clinical trial. Additionally, they should reflect only those aspects that matter to patients, because in the case of breast sensation, the patient is always right.

Supplementary Information The online version contains supplementary material available at https://doi.org/10.1007/s40271-021-00565-5.

Acknowledgements We wish to thank librarians Gregor Franssen for help constructing the search strategy and Helene Brantjes for retrieving full-text articles. We are grateful to biostatistician Sander van Kuijk for helpful suggestions on how to present the data.

\section{Declarations}

Funding This research did not receive any specific grant from funding agencies in the public, commercial, or non-profit sectors.

Conflicts of interest None of the authors has a financial interest to declare in relation to the content of this article.

Ethics approval Not applicable.

Consent to participate Not applicable.

Consent for publication Not applicable.

Availability of data and material All data analyzed during this review are included in this published article and its Online Resource files.

Code availability N/A: no code or software was used.

Author's contributions Conceptualization: SMHT, MLK. Literature search and data extraction: HPS, RCHD. Data analysis: HPS, RCHD, MLK. Writing (original draft preparation): HPS, RCHD. Writing (review and editing): MLK, SMHT, and RRWJH. Supervision: MLK, SMHT, and RRWJH.

Open Access This article is licensed under a Creative Commons Attribution-NonCommercial 4.0 International License, which permits any non-commercial use, sharing, adaptation, distribution and reproduction in any medium or format, as long as you give appropriate credit to the original author(s) and the source, provide a link to the Creative Commons licence, and indicate if changes were made. The images or other third party material in this article are included in the article's Creative Commons licence, unless indicated otherwise in a credit line to the material. If material is not included in the article's Creative Commons licence and your intended use is not permitted by statutory regulation or exceeds the permitted use, you will need to obtain permission directly from the copyright holder. To view a copy of this licence, visit http://creativecommons.org/licenses/by-nc/4.0/. 


\section{REFERENCES}

1. Lagergren J, Wickman M, Hansson P. Sensation following immediate breast reconstruction with implants. Breast $\mathbf{J}$. 2010;16(6):633-8. https://doi.org/10.1111/j.1524-4741.2010. 00984.x.

2. Shridharani S, Magarakis M, Stapleton S, Basdag B, Seal S, Rosson G. Breast sensation after breast reconstruction: A systematic review. J Reconstr Microsurg. 2010;26(5):303-10. https://doi.org/ 10.1055/s-0030-1249313.

3. Levin R, Meston C. Nipple/breast stimulation and sexual arousal in young men and women. J Sex Med. 2006;3(3):450-4. https:// doi.org/10.1111/j.1743-6109.2006.00230.x.

4. D'Alonzo M, Cipriani C. Vibrotactile sensory substitution elicits feeling of ownership of an alien hand. PLoS ONE. 2012;7(11): e50756. https://doi.org/10.1371/journal.pone.0050756.

5. Enajat M, Rozen W, Audolfsson T, Acosta R. Thermal injuries in the insensate deep inferior epigastric artery perforator flap: case series and literature review on mechanisms of injury. Microsurgery. 2009;29(3):214-7. https://doi.org/10.1002/micr.20601.

6. Jaeger M, Wagman Y, Liran A, Harats M, Winkler E, Haik J, Tessone A. A literature review of burns in reconstructed breasts after mastectomy. Wounds. 2016;28(12):422-8.

7. Mohanna P, Raveendran S, Ross D, Roblin P. Thermal injuries to autologous breast reconstructions and their donor sites-literature review and report of six cases. J Plast Reconstr Aesthet Surg. 2010;63(3):e255-60. https://doi.org/10.1016/j.bjps.2009.06.040.

8. Price R, Mokbel K, Carpenter R. Hot-water bottle induced thermal injury of the skin overlying Becker's mammary prosthesis. Breast. 1999;8(3):141-2. https://doi.org/10.1054/brst.1999.0053.

9. Freeman B. Subcutaneous mastectomy for benign breast lesions with immediate or delayed prosthetic replacement. Plast Reconstr Surg Transplant Bull. 1962;30:676-82. https://doi.org/10.1097/ 00006534-196212000-00008.

10. Slezak S, McGibbon B, Dellon A. The sensational transverse rectus abdominis musculocutaneous (TRAM) flap: return of sensibility after TRAM breast reconstruction. Ann Plast Surg. 1992;28(3):210-7. https://doi.org/10.1097/00000637-19920 3000-00004.

11. Lindau S, Bensmaia S. Using bionics to restore sensation to reconstructed breasts. Front Neurorobot. 2020;14:24. https://doi.org/10. 3389/fnbot.2020.00024.

12. Rolke R, Baron R, Maier C, Tölle T, Treede D, Beyer A, Binder A, Birbaumer N, Birklein F, Bötefür I, Braune S, Flor H, Huge V, Klug R, Landwehrmeyer G, Magerl W, Maihöfner C, Rolko C, Schaub C, Scherens A, Sprenger T, Valet M, Wasserka B. Quantitative sensory testing in the German Research Network on Neuropathic Pain (DFNS): standardized protocol and reference values. Pain. 2006;123(3):231-43. https://doi.org/10.1016/j.pain. 2006.01.041.

13. Mercieca-Bebber R, King M, Calvert M, Stockler M, Friedlander $M$. The importance of patient-reported outcomes in clinical trials and strategies for future optimization. Patient Relat Outcome Meas. 2018;9:353-67. https://doi.org/10.2147/PROM.S156279.

14. Vartanian E, Lo A, Hershenhouse K, Jacob L, Patel K. The role of neurotization in autologous breast reconstruction: can reconstruction restore breast sensation? J Surg Oncol. 2021;123(5):1215-31. https://doi.org/10.1002/jso.26422.

15. Weissler J, Koltz P, Carney M, Serletti J, Wu L. Sifting through the evidence: A comprehensive review and analysis of neurotization in breast reconstruction. Plast Reconstr Surg. 2018;141(3):550 65. https://doi.org/10.1097/PRS.0000000000004108.

16. Beugels J, Cornelissen A, Spiegel A, Heuts E, Piatkowski A, Van der Hulst R, Tuinder S. Sensory recovery of the breast after innervated and non-innervated autologous breast reconstructions: A systematic review. J Plast Reconstr Aesthet Surg. 2017;70(9):1229-41. https://doi.org/10.1016/j.bjps.2017.05.001.

17. Moher D, Liberati A, Tetzlaff J, Altman D, Group P. Preferred reporting items for systematic reviews and meta-analyses: the PRISMA statement. BMJ. 2009;339:b2535. https://doi.org/10. 1136/bmj.b2535.

18. Aminoff M. Numbness, tingling, and sensory loss. In: Jameson JL, Fauci AS, Kasper DL, Hauser SL, Longo DL, Loscalzo J, editors. Harrison's principles of internal medicine. 20th ed. New York: McGraw-Hill; 2018.

19. Bramer W, de Jonge G, Rethlefsen M, Mast F, Kleijnen J. A systematic approach to searching: an efficient and complete method to develop literature searches. J Med Libr Assoc. 2018;106(4):53141. https://doi.org/10.5195/jmla.2018.283.

20. Rothrock N, Kaiser K, Cella D. Developing a valid patientreported outcome measure. Clin Pharmacol Ther. 2011;90(5):73742. https://doi.org/10.1038/clpt.2011.195.

21. Mokkink L, Prinsen C, Patrick D, Alonso J, Bouter L, De Vet H, Terwee C (2018) COSMIN methodology for systematic reviews of patient-reported outcome measures (PROMs): User manual. https://www.cosmin.nl/wp-content/uploads/COSMIN-systreview-for-PROMs-manual_version-1_feb-2018.pdf. Accessed 9 Mar 2021.

22. Kolko J. Exposing the magic of design. New York: Oxford University Press; 2011.

23. Aygin D, Cengiz H. Life quality of patients who underwent breast reconstruction after prophylactic mastectomy: systematic review. Breast Cancer. 2018;25(5):497-505. https://doi.org/10.1007/ s12282-018-0862-8.

24. di Summa P, Oranges C, Watfa W, Sapino G, Keller N, Sherylin T, Ben C, Schaefer D, Raffoul W. Systematic review of outcomes and complications in nonimplant-based mastopexy surgery. J Plast Reconstr Aesthet Surg. 2019;72(2):243-72. https://doi.org/10. 1016/j.bjps.2018.10.018.

25. Ducic I, Zakaria H, Felder J 3rd, Fantus S. Nerve injuries in aesthetic breast surgery: Systematic review and treatment options. Aesthet Surg J. 2014;34(6):841-56. https://doi.org/10.1177/10908 20X14536726.

26. Razdan S, Patel V, Jewell S, McCarthy C. Quality of life among patients after bilateral prophylactic mastectomy: a systematic review of patient-reported outcomes. Qual Life Res. 2016;25(6):1409-21. https://doi.org/10.1007/s11136-015-1181-6.

27. Satteson E, Brown B, Nahabedian M. Nipple-areolar complex reconstruction and patient satisfaction: a systematic review and meta-analysis. Gland Surg. 2017;6(1):4-13. https://doi.org/10. 21037/gs.2016.08.01.

28. Blondeel P, Demuynck M, Mete D, Monstrey S, Van Landuyt K, Matton G, Vanderstraeten G. Sensory nerve repair in perforator flaps for autologous breast reconstruction: sensational or senseless? Br J Plast Surg. 1999;52(1):37-44. https://doi.org/10.1054/ bjps.1998.3011.

29. Cornelissen A, Beugels J, van Kuijk S, Heuts E, Rozen S, Spiegel A, Van der Hulst R, Tuinder S. Sensation of the autologous reconstructed breast improves quality of life: A pilot study. Breast Cancer Res Treat. 2018;167(3):687-95. https://doi.org/10.1007/ s10549-017-4547-3.

30. Isenberg J. Sense and sensibility: Breast reconstruction with innervated TRAM flaps. J Reconstr Microsurg. 2002;18(1):23-8. https://doi.org/10.1055/s-2002-19705.

31. Magarakis M, Venkat R, Dellon A, Shridharani S, Bellamy J, Vaca E, Jeter S, Zoras O, Manahan M, Rosson G. Pilot study of breast sensation after breast reconstruction: evaluating the effects of radiation therapy and perforator flap neurotization on sensory 
recovery. Microsurgery. 2013;33(6):421-31. https://doi.org/10. 1002/micr.22124.

32. Puonti H, Jaaskelainen S, Hallikainen H, Partanen T. A new approach to microneurovascular TRAM-flap breast reconstruction-a pilot study. J Plast Reconstr Aesthet Surg. 2011;64(3):346-52. https://doi.org/10.1016/j.bjps.2010.05.011.

33. Andersen K, Christensen K, Kehlet H, Bidstup P. The effect of pain on physical functioning after breast cancer treatment: development and validation of an assessment tool. Clin J Pain. 2015;31(9):794-802. https://doi.org/10.1097/AJP.0000000000 000156.

34. Baron R, Kelvin J, Bookbinder M, Cramer L, Borgen P, Thaler H. Patients' sensations after breast cancer surgery. A pilot study. Cancer Pract. 2000;8(5):215-22. https://doi.org/10.1046/j.15235394.2000.85005.x.

35. Didier F, Radice D, Gandini S, Bedolis R, Rotmensz N, Maldifassi A, Santillo B, Luini A, Galimberti V, Scaffidi E, Lupo F, Martella $\mathrm{S}$, Petit J. Does nipple preservation in mastectomy improve satisfaction with cosmetic results, psychological adjustment, body image and sexuality? Breast Cancer Res Treat. 2009;118(3):62333. https://doi.org/10.1007/s10549-008-0238-4.

36. Stanton A, Krishnan L, Collins C. Form or function? Part 1. Subjective cosmetic and functional correlates of quality of life in women treated with breast-conserving surgical procedures and radiotherapy. Cancer. 2001;91(12):2273-81.

37. Brown A, Hill C, Khan K. Outcome of reduction mammaplasty A patients' perspective. Br J Plast Surg. 2000;53(7):584-7. https:// doi.org/10.1054/bjps.2000.3380.

38. Dahlback C, Manjer J, Rehn M, Ringberg A. Determinants for patient satisfaction regarding aesthetic outcome and skin sensitivity after breast-conserving surgery. World J Surg Oncol. 2016;14(1):303. https://doi.org/10.1186/s12957-016-1053-8.

39. Dossett L, Lowe J, Sun W, Lee M, Smith P, Jacobsen P, Laronga C. Prospective evaluation of skin and nipple-areola sensation and patient satisfaction after nipple-sparing mastectomy. J Surg Oncol. 2016;114(1):11-6. https://doi.org/10.1002/jso.24264.

40. Hart A, Pinell-White X, Losken A. The psychosexual impact of postmastectomy breast reconstruction. Ann Plast Surg. 2016;77(5):517-22. https://doi.org/10.1097/SAP.0000000000 000665 .

41. Casaubon J, Kuehn R, Pesek S, Raker C, Edmonson D, Stuckey A, Gass J. Breast-specific sensuality and appearance satisfaction: comparison of breast-conserving surgery and nipple-sparing mastectomy. J Am Coll Surg. 2020;230(6):990-8. https://doi.org/10. 1016/j.jamcollsurg.2020.02.048.

42. Lee C, Pignone M, Deal A, Blizard L, Hunt C, Huh R, Liu Y, Ubel P. Accuracy of predictions of patients with breast cancer of future well-being after immediate breast reconstruction. JAMA Surg. 2018;153(4): e176112. https://doi.org/10.1001/jamasurg. 2017.6112 .

43. Smoll N, Marne B, Maung H, Findlay M, Hunter-Smith D. Nipple sensation losses in Hall-Findlay breast reduction technique. Plast Reconstr Surg. 2013;131(3):461e-e462. https://doi.org/10.1097/ PRS.0b013e31827c739f.

44. Spear S, Schaffner A, Jespersen M, Goldstein J. Donor-site morbidity and patient satisfaction using a composite nipple graft for unilateral nipple reconstruction in the radiated and nonradiated breast. Plast Reconstr Surg. 2011;127:1437-46. https://doi.org/ 10.1097/PRS.0b013e318208d107.

45. Unukovych D, Johansson H, Johansson E, Arver B, Liljegren A, Brandberg Y. Physical therapy after prophylactic mastectomy with breast reconstruction: a prospective randomized study. Breast. 2014;23(4):357-63. https://doi.org/10.1016/j.breast.2014.01.010.

46. Lagergren J, Edsander-Nord A, Wickman M, Hansson P. Longterm sensibility following nonautologous, immediate breast reconstruction. Breast J. 2007;13(4):346-51. https://doi.org/10. 1111/j.1524-4741.2007.00441.x.

47. Davis Sears E, Chung K. A guide to interpreting a study of patientreported outcomes. Plast Reconstr Surg. 2012;129(5):1200-7. https://doi.org/10.1097/PRS.0b013e31824a2e21.

48. Brennan W, Haiavy J. Transumbilical breast augmentation: a practical review of a growing technique. Ann Plast Surg. 2007;59(3):243-9. https://doi.org/10.1097/SAP.0b013e3180 $30264 f$.

49. Pusic A, Chen C, Cano S, Klassen A, McCarthey C, Collins D, Cordeiro P. Measuring quality of life in cosmetic and reconstructive breast surgery: a systematic review of patient-reported outcomes instruments. Plast Reconstr Surg. 2007;120(4):823-37. https://doi.org/10.1097/01.prs.0000278162.82906.81.

50. Reese J, Porter L, Casale K, Bantug E, Bober S, Schwartz S, Smith K. Adapting a couple-based intimacy enhancement intervention to breast cancer: a developmental study. Health Psychol. 2016;35(10):1085-96. https://doi.org/10.1037/hea0000413.

51. Harcourt D, Rumsey N. Mastectomy patients' decision-making for or against immediate breast reconstruction. Psychooncology. 2004;13(2):106-15. https://doi.org/10.1002/pon.711.

52. Bebbington Hatcher M, Fallowfield L. A qualitative study looking at the psychosocial implications of bilateral prophylactic mastectomy. Breast. 2003;12(1):1-9.

53. Klassen A, Pusic A, Scott A, Klok J, Cano S. Satisfaction and quality of life in women who undergo breast surgery: a qualitative study. BMC Womens Health. 2009;9:11. https://doi.org/10.1186/ 1472-6874-9-11.

54. Lehmann C, Gumener R, Montandon D. Sensibility and cutaneous reinnervation after breast reconstruction with musculocutaneous flaps. Ann Plast Surg. 1991;26(4):325-7. https://doi.org/10.1097/ 00000637-199104000-00006.

55. Matthews H, Turner A, Williamson I, Clyne W. "It's a silver lining": A template analysis of satisfaction and quality of life following post-mastectomy breast reconstruction. Br J Health Psychol. 2018;23(2):455-75. https://doi.org/10.1111/bjhp.12299.

56. Schmidt J, Wetzel C, Lange K, Heine N, Ortmann O. Patients' experience of breast reconstruction after mastectomy and its influence on postoperative satisfaction. Arch Gynecol Obstet. 2017;296(4):827-34. https://doi.org/10.1007/s00404-017-4495-5.

57. Snell L, McCarthy C, Klassen A, Cano S, Rubin L, Hurley K, Montgomery G, Cordeiro P, Pusic A. Clarifying the expectations of patients undergoing implant breast reconstruction: a qualitative study. Plast Reconstr Surg. 2010;126(6):1825-30. https://doi.org/ 10.1097/PRS.0b013e3181f44580.

58. Glassey R, O'Connor M, Ives A, Saunders C, Hardcastle S. Influences on satisfaction with reconstructed breasts and intimacy in younger women following bilateral prophylactic mastectomy: a qualitative analysis. Int J Behav Med. 2018;25(4):390-8. https:// doi.org/10.1007/s12529-018-9722-3.

59. Cornelissen A, Tuinder S, Heuts E, van der Hulst R, Slatman J. What does a breast feel like? A qualitative study among healthy women. BMC Women's Health. 2018;18(1):82. https://doi.org/ 10.1186/s12905-018-0577-1.

60. Temple C, Ross D, Kim S, Tse R, Bettger-Hahn M, Gan B, MacDermid J. Sensibility following innervated free TRAM flap for breast reconstruction: Part II. Innervation improves patient-rated quality of life. Plast Reconstr Surg. 2009;124(5):1419-25. https:// doi.org/10.1097/PRS.0b013e3181b98963.

61. Alderman A, Chung K. Discussion. Sensibility following innervated free TRAM flap for breast reconstruction: Part II. Innervation improves patient-rated quality of life. Plast Reconstr Surg. 2009;124(5):1426-8. https://doi.org/10.1097/PRS.0b013e3181 baba54.

62. Stromps J, Bozkurt A, Grieb G, Kim B, Wiezik M, Pallua N. Spontaneous reinnervation of deep inferior epigastric perforator 
flaps after delayed breast reconstruction. J Reconstr Microsurg. 2016;32(3):169-77. https://doi.org/10.1055/s-0035-1564062.

63. (2020) GLOBOCAN 2020: New Global Cancer Data. https:// www.uicc.org/news/globocan-2020-new-global-cancer-data. Accessed 18 Mar 2021.

64. Brennan M, Spillane A. Uptake and predictors of post-mastectomy reconstruction in women with breast malignancy - Systematic review. Eur J Surg Oncol. 2013;39(6):527-41. https://doi.org/10. 1016/j.ejso.2013.02.021.

65. Sung H, Ferlay J, Siegel R, Laversanne M, Soerjomataram I, Jemal A, Bray F. Global cancer statistics 2020: GLOBOCAN estimates of incidence and mortality worldwide for 36 cancers in 185 countries. CA Cancer J Clin. 2021. https://doi.org/10.3322/caac.21660.

66. Lim D, Metcalfe K, Narod S. Bilateral mastectomy in women with unilateral breast cancer: a review. JAMA Surg. 2021. https://doi. org/10.1001/jamasurg.2020.6664.
67. Cancer WHOIAfRo. WHO Cancer Mortality Database. https:// www-dep.iarc.fr/WHOdb/WHOdb.htm. Accessed 18 Mar 2021.

68. Tsangaris E, Klassen A, Kaur M, Voineskos S, Bordeleau L, Zhong T, Broyles J, Pusic A. Development and psychometric validation of the BREAST-Q sensation module for women undergoing post-mastectomy breast reconstruction. Ann Surg Oncol. 2021;28(9):5183-93. https://doi.org/10.1245/ s10434-021-10094-y.

69. Abramsohn E, Anitescu M, Flynn K, Grubb D, Hazen A, Paradise K, Pinkerton E, Schumm P, Sobecki-Rausch J, Bensmaia S, Tessler Lindau S. The bionic breast project: developing a measure of breast function in women with and without a history of breast cancer using promis standards. ISSWSH. 2020;2020:S221-2. 